\title{
Caracterização zoométrica de novilhas remanescentes da raça Pantaneira
}

\author{
Morphological characterization of heifers remaining from Pantaneira breed
}

\author{
Marcos Paulo Gonçalves de Rezende ${ }^{I^{*}}$ Dirce Ferreira Luz \\ Geovane Gonçalves Ramires ${ }^{\text {III }}$ Nicacia Monteiro de Oliveira ${ }^{\mathrm{I}}$ \\ João Antônio Barbosa Filho ${ }^{\mathrm{IV}}$ Marcus Vinicius Moraes de Oliveira ${ }^{\mathrm{I}}$
}

\begin{abstract}
- NOTA -
RESUMO

Esse trabalho apresenta valores médios, erros padrão e correlações de Pearson de 10 medidas lineares (alturas de cernelha, garupa e tórax; perímetros da canela e torácico; comprimentos da garupa e corporal; abertura de ísquio; largura da garupa; e peso corpóreo) de 14 novilhas Pantaneiras, bem como uma análise multivariada por meio de análise de componentes principais (PCA) para reduzir quantidade de variáveis passíveis de serem mantidas e utilizadas em seleção, por apresentar maior contribuição para variação total. Os animais, com idade de 36 meses, não prenhes, pertencem ao Núcleo de Bovinos Pantaneiros de Aquidauana, da Universidade Estadual de Mato Grosso do Sul. As novilhas apresentaram medidas próximas aos bovinos de pequeno porte, como as raças Jersey e Sindi, com peso médio de $375 \pm 10 \mathrm{Kg}$. O peso corpóreo apresentou correlação de magnitude moderada e significativa $(P<0,05)$ com as alturas de cernelha (55\%) e garupa (69\%) e com o comprimento corporal (59\%); e alta com perímetro torácico (82\%). Nesse sentido, ao escolher exemplares mais altos, compridos e com maior garupa e circunferência torácica, certamente se estará selecionando animais mais pesados. Com base no peso das medidas, foram necessários apenas 4 PCA com autovalor mínimo de 1,0, acumulando 83,95\% da variância acumulada. Extraiu-se 1

rump and thorax height; shin and thoracic perimeters; rump and body lengths; opening of ischium; breadth of the rump, and body weight) of 14 heifers Pantaneira breed, as well as a multivariate analysis using principal component analysis (PCA) to reduce the amount of variables that can be kept and used in selection, due to a higher contribution to the total variation. The animals, with 36 months, not pregnant, belong to Nucleus of Cattle Pantaneiros of Aquidauana, University State of Mato Grosso do Sul. The heifers presented measures near the small sized cattle, such as Sindhi and Jersey breeds, with weight of $375 \pm 9.95 \mathrm{~kg}$. The body weight showed a moderate correlation and significant $(P<0.05)$ with withers $(55 \%)$ and rump heights (69\%) and body length (59\%), and high thoracic perimeter (82\%). Therefore, by choosing the highest exemplary, of longer and greater rump and thoracic circumference, certainly will be selected the heavier animals. Based on the weight of the measures were needed only 4 PCA with minimum autovalue of 1.0, reaching $83.95 \%$ of the cumulative variance. It was extracted 1 characteristic with the highest weight in each PCA, which may be maintained in future studies or screening programs, with a reduction of $60 \%$, thus, indicating the measurements of height and breadth of the rump, thoracic perimeters and length body as being essential to select of animals phenotypically superior.
\end{abstract} característica com maior peso em cada PCA, passível de ser mantida em estudos futuros ou em programas de seleção, havendo redução de 60\%; sendo assim, indicam-se as medidas de altura e largura de garupa, perímetro torácico e comprimento corporal, como sendo essenciais para a escolha de animais fenotipicamente superiores.

Palavras-chave: Bos taurus taurus, conservação, mensuração, raça naturalizada, seleção.

\section{ABSTRACT}

This paper exhibit average values, standard error and Pearson correlations of 10 linear measurements (withers,
Key words: Bos Taurus taurus, conservation, measurement, naturalized breed, selection.

O bovino Pantaneiro é um grupo genético oriundo da miscigenação de animais Bos taurus taurus, provenientes da Península Ibérica no continente Europeu, e trazidos para o Brasil durante a colonização. Outrora considerado a base da pecuária pantaneira, com milhares de cabeças, atualmente,

IPrograma de Pós-graduação em Zootecnia Produção Animal no Cerrado/Pantanal, Universidade Estadual do Mato Grosso do Sul (UEMS),

CP 67, 79200-000, Aquidauana, MS, Brasil. E-mail: marcos_re_z@hotmail.com. *Autor para correspondência.

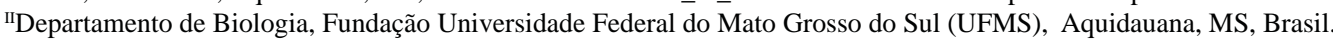

IIIUEMS, Aquidauana, MS, Brasil.

${ }^{\text {IV}}$ Programa de Pós-graduação em Ciência Animal, Fundação Universidade Estadual de Londrina (UEL), Londrina, PR, Brasil. Recebido 13.05.13 Aprovado 01.11.13 Devolvido pelo autor 05.02.14 CR-2013-0661.R1 
encontra-se em risco de extinção, com menos de 500 indivíduos puros manejados pelo homem e de mais algumas dezenas mantidos em estado feral.

As condições de elevada temperatura ambiental, grandes variações pluviométricas e menor qualidade nutricional das forrageiras promoveram, através da seleção natural, a formação de uma nova raça geneticamente adaptada às condições do Pantanal e genuinamente brasileira (SANTOS et al., 2005). Nesse contexto, vários estudos (MAZZA et al., 1992; JULIANO et al., 2011) vêm abordando a importância da valorização dessas raças naturalizadas, divulgando que, além do aspecto adaptativo, elas podem, através da seleção e do melhoramento genético, demonstrar fenótipos com bons índices zootécnicos.

HAGGER \& HOFER (1991) sugerem, para a seleção e melhoramento genético de uma determinada raça, uma análise criteriosa das medidas corporais, devido à facilidade de tomá-las em larga escala e ao fato de não oscilarem dentro de determinado período. YAKUBU et al. (2011) citam que a análise de variância e a correlação são amplamente utilizadas para caracterizar relações fenotípicas e genéticas nos programas de melhoramento e conservação genética. CRUZ \& REGAZZI (2001) atribuem ainda a análise multivariada como uma ferramenta interessante para redução de características a serem usadas em estudos posteriores.

Assim, objetivou-se divulgar valores médios e correlações de Pearson de 10 medidas lineares mensuradas em novilhas da raça Pantaneira, bem como realizar análises multivariadas por meio de análise de componentes principais (PCA), com o intuito de reduzir a quantidade de variáveis passíveis de serem mantidas e fundamentais para a seleção dos animais, por apresentarem maior contribuição para variação total.

Com auxílio de fita métrica, hipômetro e balança mecânica e utilizando-se da metodologia proposta por BIACHINI et al. (2006), mensurou-se em 14 novilhas da raça Pantaneira as medidas de altura cernelha (AC), altura de garupa (AG), altura de tórax (AT), perímetro de canela (PC), perímetro torácico (PT), comprimento de garupa (CG), comprimento corporal (CC), abertura de ísquio (IS), largura de garupa (LG) e o peso corpóreo (PCp). Os animais, com idade de 36 meses e não acasalados, pertencem ao Núcleo in situ de Bovinos Pantaneiros de Aquidauana (NUBOPAN) da Universidade Estadual de Mato Grosso do Sul / Unidade Universitária de Aquidauana (UEMS/UUA).

Utilizando o programa Bioestat 5.0, estimaram-se as médias, erros padrão e coeficientes de variação (CV). As associações das medidas lineares foram realizadas através da análise de correlação de
Pearson; e a análise multivariada foi estimada através da análise de componentes principais (PCA), a qual foi determinada utilizando-se o critério de Kaiser com base no autovalor, estabelecendo um valor mínimo de 1 e acúmulo de pelo menos $70 \%$ da variação explicada (CRUZ et al., 2004).

As médias, em centímetros, e os respectivos erros padrão das medidas lineares mensuradas nas

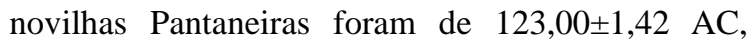

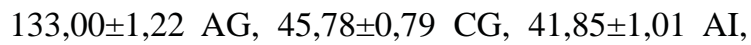
$52,85 \pm 1,30$ LG, $147,00 \pm 1,70$ PT, 18,78 $\pm 0,31$ PC, $128,00 \pm 2,41$ CC, $77,64 \pm 1,13$ AT; e de $375,00 \pm 9,95 \mathrm{~kg}$ para o PCp. Verifica-se ainda que os CV foram muito baixos, não excedendo valores superiores a 10\%, o que reflete a homogeneidade da expressão fenotípica desses animais. PEZZINI (2010) também observou resultados semelhantes ao avaliar animais da raça Crioula Lageana, um bovino naturalizado na região Sul e formado pela mistura de raças europeias, na época da colonização do Brasil.

Apesar das novilhas terem sido criadas em pastagem de melhor qualidade nutricional que seus ancestrais, verifica-se por meio das medidas lineares que a raça apresenta perfil de pequeno porte. SANTOS et al. (2002) verificaram que bovinos Pantaneiros apresentam menor capacidade produtiva em relação a raças comerciais, como a Nelore. Todavia, de acordo com RANGEL et al. (2004) apesar dessa menor produtividade, deve-se considerar que são produtos gerados sobre influência direta de três séculos da seleção natural no Pantanal, não possuindo em seu genótipo nenhuma influência de melhoramento genético para interesse econômico.

WOOLLIAMS et al. (1986) reforçam que muitas dessas populações naturalizadas podem conter alelos que conferem resistência às doenças; além de poderem expressar melhor seu potencial genético em condições ambientais menos favoráveis. Corroborando, DANI et al. (2012) indicaram que a característica thrifty tem sido observada em bovinos portadores do alelo selvagem da região promotora do gen do hormônio do crescimento, G1, sendo estes animais capazes de sobreviver adequadamente em condições de restrição nutricional típicas da vegetação nativa, o que não ocorre com os bovinos portadores do alelo comum ou G2.

Considerando que o sistema de manejo do gado no Pantanal é extensivo, baseado principalmente na cria de bezerros, ABREU et al. (2007) reportaram que a idade ao primeiro parto é reflexo direto da curva de crescimento e, como as matrizes Pantaneiras possuem perfil corporal pequeno, a curva de crescimento dos animais é consequentemente 
menor, sendo porém bem adequada para a região do Pantanal. Outro fator é a menor exigência nutricional, pois, segundo ROCHA et al. (2003) a definição da necessidade de nutrientes do animal é estabelecida em função do seu porte físico.

Observou-se maior correlação entre AC/ AG (84\%) e PCp/PT (82\%), sendo ambos classificados como casos de magnitude alta, no sentido positivo, sendo significativas $(\mathrm{P}<0,05)$; e, semelhante ao observado por CONTRERAS et al. (2011) ao estudar vacas Criollo Limonero na Venezuela, o que indica possibilidade de resposta correlacionada entre essas medidas. PRAJAPATI et al. (1991) retratam que o PT é altamente correlacionado com o PCp, acompanhando ainda suas flutuações periódicas devido a influências ambientais.

Exceto para AC/AG (r: 0,84), AC/PC (r: 0,54), CC/AC (r: 0,56), AC/PCp (r: 0,55), AG/PC (r: 0,56), CC/AG (r: 0,56), AG/AT (r: 0,56), AG/ PCp (r: 0,69), LG/CC (r: 0,71), PT/PCp (r: 0,82) e PC/CC (r: 0,59), todas os demais pares de medidas não apresentaram diferença significativa e, no geral, com tendência de correlação com magnitude média a baixa $(\mathrm{P}<0,05)$, divergindo de HAGGER \& HOFER (1991) e PRAJAPATI et al. (1991). O PCp apresentou correlação de magnitude moderada a alta com AC, AG, PT e CC e, considerando resultados de PEZZINI (2010) e ROCHA et al. (2003), se selecionar os exemplares mais altos, compridos e de maior garupa, bem como maior PT, certamente serão selecionados animais mais pesados dentro do rebanho.

Com base no peso das 10 medidas lineares, determinaram-se aquelas de maior importância, de acordo com cada PCA, bem como a ordenação das variáveis do primeiro ao último PCA. Para as novilhas, foram necessários apenas 4 PCA com autovalor mínimo de 1 , acumulando $83,95 \%$ da variância acumulada. Outro estudo com animais domésticos apresentou redução de 15 variáveis por meio da análise dos PCA (PINTO et al., 2005).

A extração de 1 característica, com maior peso em cada PCA, permitiu a redução de $60 \%$ das variáveis, quando as medidas de AG, LG, PT e CC foram passíveis de ser mantidas em estudos futuros, bem como procedimentos de seleção. Assim, infere-se que o presente trabalho registrou novilhas com baixo perfil corporal, medidas lineares com respostas correlacionadas e selecionou, através da análise multivariada, as medidas com maior contribuição para variação total.

\section{REFERÊNCIAS}

ABREU, U.G.P. et al. Caracterização fenotípica e genética da precocidade sexual do bovino Pantaneiro. Archivos de Zootecnia, v.56, sup.1, p.627-631, 2007. Disponível em: <http://www.uco.es/ organiza/servicios/publica/az/php/az.php?idioma_global=0\&revis ta=135\&codigo=1502>. Acesso em: 05 dez. 2012.

BIACHINI, E. et al. Características corporais associadas com a adaptação ao calor em bovinos naturalizados brasileiros. Pesquisa Agropecuária Brasileira, v.41, n.9, p.1443-1448, 2006. Disponível em: <http://seer.sct.embrapa.br/index.php/pab/article/ view/7301>. Acesso em: 05 dez. 2012. Doi: 10.1590/S0100204X2006000900014.

CONTRERAS, G. et al. Caracterización morfológica e 16 índices zoométricos de vacas CriolloLimonero de Venezuela. Revista de La Facultad de Agronomía, v.28, p.91-103, 2011. Disponível em: <http://biblioteca.universia.net/html_bura/ficha/params/title/ caracterizacion-morfologica-indices-zoometricos-vacas-criollolimonero-venezuela/id/53657958.html>. Acesso em: 05 dez. 2012.

CRUZ, C.D. et al. Modelos biométricos aplicados ao melhoramento genético. 3.ed. Viçosa: UFV, 2004. 480p.

CRUZ, C.D.; REGAZZI, A.J. Modelos biométricos aplicados ao melhoramento genético. 2.ed. Viçosa: UFV, 2001. 390p.

DANI, M.A. et al. Synergistic and threshold effects of GH1 and GHR promoter size variation on body growth and fat accrual in young Nelore (Bos indicus) bulls. Genetics and Molecular Research, v.11, p.1783-98, 2012. Disponível em: <http://www. ncbi.nlm.nih.gov/pubmed/22843055>. Acesso em: 05 dez. 2012. Doi: $10.4238 / 2012$.

HAGGER, C.; HOFFER, A. Phenotypic and genetic relationships between wither height, heart girth and milk yield in the Swiss Braunvieh and Simmental breeds. Livestock Production Science, v.28, n.3, p.265-71, 1991. Disponível em:<http://www. sciencedirect.com/science/article/pii/030162269190147I> Acesso em: 05 dez. 2012. Doi: 10.1016/0301-6226(91)90147-I

JULIANO, R.S. et al. Análise de características reprodutivas indicadoras de puberdade em tourinhos Pantaneiro. Archivos de Zootecnia, v.60, n.231, p.325-3208, 2011. Disponível em: <http:// www.uco.es/organiza/servicios/publica/az/php/az.php?idioma_glo bal=0\&revista=164\&codigo=1925> Acesso em: 05 dez. 2012.

MAZZA, M.C.M. et al. Phenotipical characterization of Pantaneiro cattle in Brasil. Archivos de Zootecnia, v.41, n.154 (extra), p.477-484, 1992. Disponível em: <http://www. uco.es/organiza/servicios/publica/az/php/az.php?idioma_ global=0\&revista=31\&codigo=496> Acesso em: 05 dez 2012 .

PEZZINI, T.G. Análise da estrutura genética, da biometria e da viabilidade populacional da raça bovina Crioula Lageana. 2010. 93f. Tese (Doutorado em Ciência Animal) - Universidade de Brasília-DF.

PINTO,L.F.B.etal.Análisemultivariadadasmedidasmorfométricas de potros da raça Mangalarga Machador: análise discriminante. Revista Brasileira de Zootecnia, v.34, n.2, p.600-612, 2005. Disponível em: <http://www.scielo.br/scielo.php?script=sci_artte xt\&pid=S1516-35982005000200030 > . Acesso em: 05 dez. 2012. Doi: 10.1590/S1516-35982005000200030.

PRAJAPATI, K.B. et al. Relationship between different measures of body size in Mehsana buffaloes. Indian Journal of Animal Science, v.61, p.88-90, 1991. 
RANGEL, P.N. et al. Similaridade genética entre raças bovinas brasileiras. Pesquisa Agropecuária Brasileira, v.39, n.4, p.97100, 2004. Disponível em: <http://seer.sct.embrapa.br/index.php/ pab/article/view/6742>. Acesso em: 05 dez. 2012. Doi: 10.1590/ S0100-204X2004000100015.

ROCHA, E.D. et al. Tamanho da vaca Nelore adulta e seus efeitos no sistema de produção de gado de corte. Arquivo Brasileiro de Medicina Veterinária e Zootecnia, v.55, n.4, p.447-479, 2003. Disponível em: <http://www.scielo.br/scielo.php?pid=S010209352003000400014\&script=sci_arttext $>$. Acesso em: 05 dez. 2012. Doi: 10.1590/S0102-09352003000400014.

SANTOS, S.A. et al. Curva de crescimento de bezerros Nelores criados em pastagem nativa na sub-região Nhecolândia, Pantanal. In: REUNIÃO DA SOCIEDADE BRASILEIRA DE ZOOTECNIA, 39., 2002, Recife, PE. Anais... Recife: UFRPE: SBZ, 2002. V.39. CD-ROOM.
SANTOS, S.A. et al. Desempenho de bezerros Pantaneiros, Nelore e Cruzados criados no Pantanal, Brasil. Archivos de Zootecnia, v.54, n.206/207, p.501-508, 2005. Disponível em: <http://www. uco.es/organiza/servicios/publica/az/php/az.php?idioma_global=0 \&revista=35\&codigo=587> . Acesso em: 05 dez. 2012

WOOLLIAMS, J.A. et al. Studies on lambs from lines genetically selected for low and high copper status. 2. Incidence of hypocuprosison improved hill pasture. Animal Production, v.43, n.2, p.303-317, 1986. Disponível em: <http://www.uco.es/ organiza/servicios/publica/az/php/az.php?idioma_global=0\&revis ta=35\&codigo=587> . Acesso em: 05 dez. 2012.

YAKUBU, A. Discriminant analysis of sexual dimorphism in morphological traits of African Muscovy ducks. Archivos de Zootecnia, v.60, n.232, p.1115-1123, 2011. Disponível em: <http:// www.uco.es/organiza/servicios/publica/az/php/az.php?idioma_gl obal=0\&revista=166\&codigo=2005> . Acesso em: 05 dez. 2012. 